\title{
Chronic Helminth Infections Protect Against Allergic Diseases by Active Regulatory Processes
}

\author{
Hermelijn H. Smits • Bart Everts • Franca C. Hartgers • \\ Maria Yazdanbakhsh
}

Published online: 6 January 2010

(C) The Author(s) 2010. This article is published with open access at Springerlink.com

\begin{abstract}
Developed countries are suffering from an epidemic rise in immunologic disorders, such as allergy-related diseases and certain autoimmunities. Several studies have demonstrated a negative association between helminth infections and inflammatory diseases (eg, allergy), providing a strong case for the involvement of helminth infections in this respect. However, some studies point in the opposite direction. The discrepancy may be explained by differences in frequency, dose, time, and type of helminth. In this review, new studies are discussed that may support the concept that chronic helminth infections in particular - but not acute infections - are associated with the expression of regulatory networks necessary for downmodulating allergic immune responses to harmless antigens. Furthermore, different components of regulatory networks are highlighted, such as the role of regulatory $\mathrm{T}$ and $\mathrm{B}$ cells, modulation of dendritic cells, early innate signals from structural cells (eg, epithelial cells), and their individual contributions to protection against allergic diseases. It is of great interest to define and characterize specific helminth molecules that have profound immunomodulatory capacities as targets for therapeutic application in the treatment or prophylaxis of allergic manifestations.
\end{abstract}

Keywords Helminth · Allergy · Dendritic cell · Treg cell · IL-10 $\cdot$ B cells

\section{Introduction}

The human immune system has evolved to mount appropriate defensive responses to various dangerous pathogens

H. H. Smits $(\triangle) \cdot$ B. Everts $\cdot$ F. C. Hartgers $\cdot$ M. Yazdanbakhsh Department of Parasitology, Leiden University Medical Center, Albinusdreef 2,

2333 ZA Leiden, The Netherlands

e-mail: h.h.smits@lumc.nl while tolerating or ignoring the innocent ones. The constant presence of chronic helminth, microbial, and protozoan infections and the daily risk of acquiring food-, water-, and vector-borne infections is still present in developing countries, whereas this was largely controlled in economically developed and Westernized countries during the 20th century. In contrast, Westernized countries now face very different problems: the epidemic rise in obesity, cardiovascular diseases, metabolic disorders, and hyperinflammatory diseases. As such, alarming increases have been observed in childhood allergy (eg, rhinitis, atopic dermatitis, and allergic and nonallergic asthma [1]), inflammatory bowel diseases (eg, Crohn's disease and ulcerative colitis), and autoimmune disorders (eg, type 1 diabetes and multiple sclerosis) [2]. It has been proposed that education of the immune system by certain microbes and parasites can prevent in part the development of inflammatory diseases [3]. Reduced infections due to improved health care and personal hygiene and decreased exposure to microorganisms and their products in our immediate environment as a result of urbanization may lead to insufficient stimulation of the immune system. This would result in an altered programming of the immune system and allow uncontrolled expression of inflammatory molecules, thus explaining the rise of inflammatory diseases in Westernized countries. Indeed, a large body of epidemiologic data indicates that some infectious agents tend to control inflammatory diseases, in which parasitic worms form an important group. In particular, the interplay between helminth infections and allergic disorders has been studied in great detail. In the current review, we focus on this interaction and highlight the importance of the infection dynamics and regulatory network. Helminth-induced mechanisms not only regulate host immunity to the worms, resulting in a mutually beneficial environment for survival of both parasite and host, but may also control the development 
of allergic diseases. Finally, we focus on the benefit of these new insights for the development of novel future therapies.

\section{Do Helminths Protect Against Allergic Disease? Evidence from Population Studies}

The number of studies on associations between allergic disorders and parasitic infections is still growing. One approach that is frequently used to determine the relationship between helminths and allergy is to study general parameters for allergic reactions, such as allergen skin prick provocation tests and questionnaires (clinician assessed or self-reported). However, this does not apply to all types/ aspects of allergic disease, as physician diagnosis is required at least for (allergic or nonallergic) asthma, eczema, or rhinitis in conjunction with clinical symptoms. A systematic meta-analysis of published findings attempted to compare a large number of studies related to intestinal helminth infection and different aspects of allergic disease [4]. However, sufficient conclusions could not always be drawn because of the relatively few studies that addressed physician-diagnosed clinical symptoms. As such, studies involving intestinal helminth infections did not yield a general consensus regarding protection against different forms of allergic disease.

However, caution should be taken in not overinterpreting the general conclusions because several crucial factors that are likely to influence the relationship between helminth and allergic disease need to be taken into account [5].

\section{Timing}

The time of first infection and duration of infection are likely to be important; early and/or long-lasting (chronic) infections are more efficient in downmodulating allergic disease, whereas later infections and/or sporadic, transient infections may enhance allergic clinical symptoms [6]. For example, the effect of geohelminths on the suppression of atopy is more important early in life and may cause a fixed deviated immune phenotype that is not changed later in life, after elimination of the infection [7]. Indeed, recent studies show that maternal geohelminth infections could affect infant immunity [8, 9], raising the possibility that the immunologic effects of infection start in the fetus.

\section{Intensity}

Heavy parasite burden may induce immune suppression, whereas mild infections may promote allergic disease. In the meta-analysis, all published studies were included that reported parasitic infection in at least $1 \%$ of the available study population [4]. This led to an enormous variation in helminth intensities and species of parasitic helminths. In some endemic areas, close to $100 \%$ of the inhabitants may be infected, representing intense and chronic helminth infections, whereas in others, helminth infections can occur occasionally and in a small percentage of the population, with an intensity that is often mild. In areas with at least moderate endemicity for different species of helminth parasites, a considerable number of studies have demonstrated an inverse association between helminth infections and allergic disorders. For example, chronic infections with intestinal helminths (Ascaris, Trichuris, and hookworms) were reported to protect against allergic reactivity in populations in Venezuela, Gambia, Ethiopia, Taiwan, and Ecuador [10]. However, in areas endemic for other helminthes (eg, schistosomes or filarial), the presence of infections also seems to be associated with lower prevalences of allergies, as shown in studies in Gabon, Brazil, and Indonesia [10]. Similarly, chronic and intense Schistosoma heamatobium infections in Ghana were negatively correlated with the prevalence of atopic disease, whereas mild infections were not $[11,12]$. Population studies in areas in which helminth infection intensities are low are conducted only sporadically [13, 14]; nevertheless, these low-intensity helminth infections seem to potentiate atopic disorders. In addition, some of the travelers to endemic areas who become infected with schistosomes develop acute schistosomiasis and can suffer from fever, lung eosinophilia, and pulmonary symptoms such as cough and shortness of breath [10].

\section{Host Genetics}

The ability to induce specific host immune regulatory mechanisms may be partially determined by host genetics. Individuals who are genetically susceptible to atopic disease may be more likely to develop allergic responses to helminth and allergens and may be genetically more resistant to infection [15]. On the other hand, people in rural Africa seem to suffer less from allergies, whereas people of African ancestry who live in affluent countries have a higher prevalence and greater severity of allergic symptoms than natives of these host countries, pointing to the involvement of genetic control in allergic diseases [12].

\section{Different Helminth Parasites}

Meta-analyses showed that infections with Trichuris, hookworm, or schistosomes were primarily negatively associated with allergen skin test reactivity, whereas hookworm infections were also associated with a reduced prevalence of allergic asthma $[4,16]$. Although the sample size was too small for the meta-analysis, a similar association was found for schistosome infections and 
asthma in individual studies [17]. In contrast, in some studies, Ascaris lumbricoides infection was associated with an increased prevalence of asthma, pointing toward the importance of the species of helminths in the relationship between allergies and helminths. In addition, helminth species for which humans are not the definitive host, such as Toxocara spp, and for which chronic infection cannot be established [18, 19] also seem to potentiate atopic disorders rather than protect against them.

Important lessons can be learned from intervention studies: short-term application of antihelminth drugs $(<12$ months) in Ecuador did not change the prevalence of atopy or clinical signs of allergy (wheeze) compared with the untreated group [20]. Deworming for 12 months in a large cohort of Vietnamese schoolchildren resulted in increased allergen sensitization, but not in clinical allergies such as eczema, wheeze, or rhinitis. However, long-term treatment for intestinal helminths ( $>22$ months) in Venezuelan or Gabonese children resulted in increased allergen sensitization and skin prick test reactivity to house dust mite [21-23], supporting a direct link between chronic and intense helminth exposure and protection from allergy. To gain a better understanding of early immunologic influence and its interplay with genetic risk factors, this needs to be further addressed in large birth cohort intervention studies in helminth-endemic areas.

\section{The Helminth Paradox}

Helminths are master regulators of the host immune response, effectively minimizing immune attacks meant to expel the worms and thereby ensuring their survival in the host for years and limiting host tissue damage. They strongly induce polarized T-helper type 2 (Th2) responses, elevated serum IgE titers, and eosinophil-rich inflammation infiltrates in the tissue. However, despite this strong Th2 polarization, chronic infections do not induce clinical symptoms of allergic disease. This presents a paradox; however, further characterization of helminth-induced immune responses shows a strong regulatory network that is discussed in the context of disordered immunoregulation, as explained below.

\section{Immune Suppression}

It is postulated that chronic helminth infections can protect against allergic disease because of their profound suppression of the host immune system, leading to a general T-cell hyporesponsiveness that is facilitated by the induction of a regulatory network. Thus far, this network has been described to include the activity of regulatory $\mathrm{T}$ (Treg) and $\mathrm{B}$ cells and modulation of innate immune cells, such as macrophages, dendritic cells (DCs), and local stromal cells, resulting in an anti-inflammatory environment characterized by increased levels of interleukin (IL)-10 and transforming growth factor (TGF)- $\beta$ [24]. This hyporesponsiveness is not only directed toward parasite antigens but seems to extend to bystander antigens, such as vaccine antigens or other pathogens. For example, impaired Th1 responses were reported to tetanus toxoid immunization [25] or against influenza virus in Schistosoma- or Onchocercainfected patients [25, 26], and reduced immune responses to Bacille Calmette-Guérin (BCG) vaccination and to the cholera vaccine during intestinal helminth infection [27].

Several studies have described interactions between helminths and malarial parasites. Although the findings on malaria-parasite loads are controversial, data on malaria pathology seem more uniform, showing that helminth infection protects against renal failure and cerebral malaria [28]. Furthermore, a recent study showed higher IL-10 responses to malaria antigens in children infected with $S$. haematobium and/or different geohelminth species [29].

It is hypothesized that bystander immunoregulation by helminth infection can also control allergen-specific inflammatory responses and thereby lead to lower prevalence of allergies in helminth-infected individuals. However, control of bystander T-cell responses may depend on particular life cycle stages or the severity/intensity of an infection. Animal models offer great opportunities to dissect the interplay between pathogens such as helminths and allergic diseases. Several studies have been reported recently.

\section{Lessons from Animal Models of Helminth-Allergy Interaction}

To study the causal relationship between helminth infections and the development of allergic diseases, several groups have developed combined murine models of allergic inflammation, acute asthma, atopic dermatitis, and several different helminth species. For example, studies with rodent nematodes (eg, Heligmosomoides polygyrus) have demonstrated that infection leads to strongly reduced ovalbumindriven eosinophilic airway inflammation and immune responses to food allergens, but not decreased symptoms of atopic dermatitis [30-32]. In part of these studies, the effect was dependent on Treg cells, whereas in others, it was reversed by treatment with blocking IL-10 antibodies $[30,33]$. A similar inhibition of lung inflammation and airway hyperresponsiveness was demonstrated by Ascaris suum eggs, A. suum extract implants, or Ascaris worm products (eg, PAS-1) in an IL-10-dependent manner [34, 35] and correspondingly by infections with Nippostrongylus brasiliensis [36] or Schistosoma mansoni [37-39]. In 
part of these studies, the suppressive effect was dependent on the activity of IL-10 [36-38] or on the activity of forkhead box P3 (FoxP3) Treg cells [39].

However, a few studies showed no effect or a partial effect on airway inflammation, whereas some even found an exacerbation of allergic disease. For example, early infections with helminths such as A. suum [40] or Toxocara canis [41] potentiated airway inflammation, and infection with Trichinella spiralis increased anaphylaxis in mice [42]. The opposing effects of different parasitic helminths in murine models may suggest variations in the immune modulating capacity of distinct species. However, this paradox could also very well reflect the difference between acute and chronic stages of infection. Indeed, by exploring acute and chronic infections with $S$. mansoni, we have observed a clear dosedependent reduction in ovalbumin-specific eosinophilic airway inflammation and airway hyperresponsiveness that was mediated by IL-10 during chronic, but not acute, infection [37].

All together, it seems that helminth infections at chronic stages are associated with general immunosuppression, which can have protective effects in different allergic disease models. The exact mechanisms by which these parasites dampen allergic responses are probably multiple. The next challenge is to find out which part of the regulatory network provides the strongest and most longlasting allergen-specific inhibitory response, including the identification of the parasite antigens that are responsible for inducing this.

\section{How Helminths Modulate Immune Responses}

It is clear from a wealth of literature that both the worms and eggs of different helminths can modulate immune responses by affecting different types of innate and adaptive immune cells. Based on the evidence, a model can be put forth to explain how helminths suppress the host immune system and guarantee their own survival. In recent years, a great deal of new information has become available that explains the immune hyperresponsiveness by a complete regulatory network in which different cell types are involved. Next to polarized Th2 responses, the development of regulatory $\mathrm{T}$ and $\mathrm{B}$ cells and immune modulation of myeloid cells, such as DCs, or alternatively activated macrophages and early innate signals from epithelial cells seems to be an important element of a chronic helminth infection and could harbor putative targets that are important for inhibition of allergic diseases. The role of alternatively activated macrophages was recently discussed elsewhere [43] and is not addressed further here.
Regulatory T and B Cells

Several studies have shown that Treg cell activity (both by natural $\mathrm{CD} 4^{+} \mathrm{CD} 25^{+} \mathrm{FoxP}^{+}$and adaptive $\mathrm{CD} 4^{+} \mathrm{IL} 10^{+} \mathrm{Tr} 1$ cells) protects against allergic disease [44]. In contrast, patients with mutations in the FOXP3 gene leading to a loss in the natural Treg compartment show several immunemediated pathologies, including allergy, whereas the activity of allergen-specific Treg cells from asthmatic patients seems to be impaired [45]. Importantly, successful allergenspecific immunotherapy in humans - that which leads to a reduction in allergic symptoms - is associated with the emergence of IL-10-producing Treg cells. Simultaneously, the B-cell compartment is affected, which is evident from an increase in $\operatorname{IgG}_{4}$ and $\operatorname{IgA}$ responses and a simultaneous decrease in IgE [46]. Central to helminth-induced immune regulation of allergic responses in humans is the cytokine IL-10, which may be produced by several different cell types, including helminth-induced regulatory $\mathrm{T}$ cells [47] and B cells [48], of which the Treg cells are clearly the most studied players.

For example, in patients suffering from onchocerciasis, antigen-specific IL-10 and/or TGF- $\beta$-secreting Treg cells were found [49, 50], whereas in patients infected with Brugia malayi, increased expression of FoxP3 (specific transcription factor of natural Treg cells) was demonstrated, as was the involvement of regulatory molecules such as TGF- $\beta$ and cytotoxic T-lymphocyte antigen 4 [51]. Likewise, schistosome-infected individuals in Kenya and Gabon had higher $\mathrm{CD} 4^{+} \mathrm{CD} 25^{\text {hi }}$ and $\mathrm{CD} 4^{+} \mathrm{CD} 25^{\mathrm{hi}}$ FoxP3 T-cell levels compared with uninfected individuals [52]. Importantly, $\mathrm{CD} 4^{+} \mathrm{CD} 25^{\mathrm{hi}}$ FoxP3 Treg cells have a clear immunologic impact on host immunity, as in vitro removal of $\mathrm{CD}^{+}{ }^{+} \mathrm{FoxP} 3$ $\mathrm{T}$ cells from peripheral blood mononuclear cells of either geohelminth- or schistosome-infected schoolchildren restored BCG-specific proliferation and interferon- $\gamma$ production [53], a phenomenon that was not observed in endemic controls.

In animal models, chronic nematode infection increased the number of $\mathrm{FoxP}^{+} \mathrm{T}$ cells [24], while in chronic schistosome infections, some studies noted increases in FoxP $3^{+} \mathrm{T}$ cells [24], whereas others did not [54]. Nevertheless, the frequency of CD103 on Treg cells increased during disease progression [54], suggesting that these cells become more active during chronic schistosomiasis [55].

Elevated IL-10 has been reported in many human and murine studies in the context of helminth infections, but the source and role of IL-10 seem to vary in response to different helminth species. In some experimental models, the high IL-10 levels were attributed to adaptive Treg cells $[30,31,56,57]$, whereas in others, IL-10 has been linked to non-Treg populations $[38,54,58]$. By using IL-10 deficient mice and by adoptive transfer of different cell subsets, it was shown that both T-cell and non-T-cell- 
derived IL-10 play an essential part in helminth-induced immune modulation $[56,58,59]$. One important alternative source of IL-10 could be the B cell.

B cells possess a variety of immune functions, including production of antibodies, presentation of antigens, and production of cytokines. A large body of literature-in particular on models of autoimmunity, but also on transplantation and cancer-shows that IL-10-producing B cells have great potential to regulate T-cell-mediated inflammatory responses and therefore are named regulatory $B$ cells $[60,61]$. For example, IL-10-producing regulatory B cells were shown to downmodulate experimental autoimmune encephalomyelitis, collagen-induced arthritis, and inflammatory bowel disease [60]. In addition, in models of chronic parasitic inflammation, such as chronic schistosomiasis and filariasis, IL-10-producing B cells were reported $[37,62,63]$ and associated with reduced pathology and modulation of T-cell responses [64, 65].

Thus far, most evidence comes from murine models and needs to be confirmed in humans. Nevertheless, a few studies have focused on B cells in human populations. For example, helminth infection in multiple sclerosis patients was associated with reduced disease activity; the B cells from these patients displayed enhanced production of IL10 , in particular a CD1 $\mathrm{d}^{\text {hi }}$ subset that was correlated with in vitro inhibition of T-cell proliferation [66]. Similarly, we found increased circulating IL-10-producing CD1d ${ }^{\text {hi }}$ B cells in S. haematobium-infected Gabonese patients.

Although the concept that regulatory B cells can dampen allergic inflammation still needs to be established in particular in humans, a small number of allergy studies in murine models have already provided supportive evidence. For example, B cells were responsible for the induction of local inhalation tolerance by inducing Treg cells during continuous allergen exposure [67]. In addition, transfer of schistosome-induced, IL-10-producing B cells strongly reduced inflammation in different models of allergic inflammation $[37,38,63]$. Interestingly, in $H$. polygyrusinfected, allergen-sensitized mice, mesenteric lymph node $\mathrm{CD} 23^{\text {hi }} \mathrm{B}$ cells transferred protection against allergic airway inflammation in an IL-10-independent manner [68]. Future studies must dissect the role of the different (regulatory) B-cell subsets in immune suppression and protection against allergic disease.

\section{Immune Modulation of Dendritic Cells by Helminth Products}

DCs residing in the mucosal lining of various peripheral tissues are central to the generation and polarization of adaptive immune responses. The main functions of DCs are to patrol the environment for possible danger signals, immediately activate local innate immune cells, and subsequently initiate appropriate adaptive immune responses in which the function and cytokine production of lymphocytes are completely determined by instructions from the DCs. DCs-but also other innate immune or resident tissue cells - are ultimately equipped to recognize a great variety of pathogen-associated molecular patterns (PAMPs) or danger-associated molecular patterns by means of various families of pattern-recognition receptors, of which the families of the Toll-like receptors (TLRs), NOD-like receptors, RIG-like receptors, and the C-type lectin receptors (CLRs) are presently the best known. As a consequence of ligation of different pattern-recognition receptors, DCs will receive signals that are subsequently translated into different sets of Th1-, Th2-, Th17- or Tregpolarizing molecules [69].

Thus far, a few signature molecules of various helminths have been identified that can modulate DCs to drive strong Th2 or Treg cell responses, a hallmark of helminth infections. The most prominent example is omega-1, a glycoprotein derived from schistosome eggs that specifically primes DCs to drive polarized $\mathrm{Th} 2$ responses $[70,71]$. Its target receptor or signaling route is currently unknown, although it has been suggested that its ribonuclease activity is involved in its capacity to condition Th2-priming DCs [71]. Another clear example is ES-62, a secreted phosphorylcholine-containing glycoprotein of the filarial nematode Acanthocheilonema viteae, as it conditions DCs to induce Th2 responses via activation of TLR4, as it is primarily mediated by its phosphorylcholine moiety [72]. Also, lacto-N-fucopentaose III (LNFPIII)/Le ${ }^{\mathrm{x}}$, a glycoconjugate carrying a carbohydrate structure found in schistosomes, has been implicated in TLR4-dependent priming of Th2 responses via DCs [73]. However, the significance of this latter finding remains controversial, as schistosomesoluble egg antigens (SEAs) harboring this same $\mathrm{Le}^{\mathrm{x}}$ motif have been shown not to bind to TLR4 $[74,75]$ and are quite capable of modulating DCs for Th2 priming in the absence of TLR signaling [76]. Ligation of TLR2 by helminth molecules seems to be more important for the conditioning of DCs to drive regulatory responses. Monoacetylated phosphatidyl serine lipids from schistosomes specifically instructed DCs to preferentially induce IL-10-producing Treg in a TLR2-dependent fashion [77], and although less potent, phosphatidylserine lipids derived from Ascaris worms had a similar effect [75]. Likewise, a recent report described the induction of Treg cells by Schistosoma japonicum HSP60-derived, peptide-treated APC in a TLR2-dependent manner [78]. Recent reports even cite a clear link between Treg cells and TLR2, as TLR2-deficient mice showed a reduced number of $\mathrm{CD} 4{ }^{+} \mathrm{CD} 25^{+}$Treg cells and immunopathology during schistosomiasis that was controlled by TLR2-primed Treg cells [79]. Interesting in this respect is the observation that Treg cells can respond 
directly to TLR2 ligands, such as Pam3Cys, but not to ligands of other TLR receptors, and this augments Treg cell proliferation $[80,81]$.

Apart from TLRs, a growing body of evidence indicates that CLRs - recognizing sugar moieties - also play an important role in the sensing of helminth glycans by DCs. The glycoproteins from SEAs are recognized and internalized by human DCs in a DC-SIGN (specific intercellular adhesion molecule-3-grabbing nonintegrin)-, mannose receptor-, and macrophage galactose-type lection-dependent manner [74, 82]. The binding of SEAs to DC-SIGN was found to be dependent on sugar motifs $\mathrm{Le}^{\mathrm{x}}$ and LDN-F, while chemical modification of the glycans present in SEAs abolished the Th2-driving capacity of SEAs [83]. This, together with the observation that $\mathrm{Le}^{\mathrm{x}}$-containing LNFPIII favors Th2-biased responses [84] strongly suggests that CLRs play a dominant role in conditioning DCs for induction of Th2 responses by schistosomal antigens. Moreover, antigens from T. canis have been recognized by DC-SIGN expressed on DCs [85], whereas the induction of a Th2 response in vivo by antigens of the parasitic nematode B. malayi as well as the free-living nematode Caenorhabditis elegans was found to be dependent on intact glycans [86]. This suggests that Th2 biasing by helminth glycans is a general phenomenon and that helminth glycans may serve as a conserved molecular pattern that instructs DCs via CLRs to drive Th2-polarized responses.

Many helminth products show the inability to classically activate DCs, as exemplified by a mixture of high molecular weight components from $A$. suum that reduced the expression of major histocompatibility complex (MHC)-II, CD80, CD86, and CD40 molecules on mouse CD11 $\mathrm{c}^{+} \mathrm{DC}$, leaving the cells hampered to support strong T-cell proliferative responses in vitro. The inhibitory effect was abolished in IL10-deficient mice [87]. Similarly, SEAs also lack the capacity to classically activate DCs [88]. As such, the concept has been put forth that helminth products actually potently suppress TLR-mediated DC activation by other microbial PAMPs. This was confirmed by many studies showing the inhibitory effects of helminth-derived components on TLR-induced activation as determined by inhibition of proinflammatory cytokine production and expression of MHC-II/costimulatory molecules [89]. The pathways underlying this suppression are still poorly understood. Interestingly, the suppression of TLR-mediated responses by helminth antigens has striking similarities to the effects induced by several microbial pathogens that target DC-SIGN [90].

Overall there is a consistent picture that helminth products, regardless of whether they interact with TLRs or CLRs, fail to induce conventional DC maturation [91] and inhibit DC activation induced by proinflammatory PAMPs, which could impair Th1 development and bias the immune response toward $\mathrm{Th} 2$ or regulatory responses.
Early Innate Signals from Neighboring Cells

Recent studies in mice with repeated airway delivery of native allergens or respiratory viruses have highlighted the critical role of innate signaling in the generation of allergic pulmonary inflammation, which shows that the complex immunologic response not only includes Th2 cell-driven inflammation, but also the participation of Th1, Th2, Th17, natural killer T cells, and airway epithelial cells (ECs). ECs in particular can influence the functions of local immune cells via the expression of a wide range of molecules, including MHC-I and MHC-II, costimulatory molecules, chemokines, cytokines, and prostaglandins. During the past few years, a particular interest has been growing in three novel EC-derived cytokines, thymic stromal lymphopoietin (TSLP), IL-25, and IL-33, and their ability to influence innate and adaptive immunity associated with Th2 cytokine-mediated inflammation at mucosal sites $[92,93]$. TSLP affects DCs, T cells, natural killer T cells, and mast cells. IL-25 primarily influences macrophages, Th2 cells, eosinophils, and mast cells. IL-33 acts on T cells, mast cells, eosinophils, and basophils, illustrating the broad role of these cytokines in the regulation of inflammatory/allergic processes [93]. However, these cytokines seem to have dual functions, as they are involved in the development of protective Th2 cytokine responses in the context of helminth infections and strongly promote pathologic responses in cases of allergic inflammation [94]. In view of the hypothesis that certain helminth infections may protect against allergic diseases, it seems contradictory that helminths also induce these strong allergic inflammationinducing cytokines. However, the location and timing may be important factors. For example, TSLP expression in the skin and lung has been linked to pathologic Th2 cytokinemediated responses, whereas TSLP expression in the intestine seems to play an important role in host protective immunity, as TSLPR-deficient mice challenged with Trichuris failed to clear worms after infection [95]. However, TSLP does not seem to be essential to every intestinal helminth because the development of protective $\mathrm{Th} 2$ immune responses after infection with $H$. polygyrus and $N$. brasiliensis was still intact in $\mathrm{TSLPR}^{-/}$mice [96]. Similarly, using the same knockout mice, it was found that TSLPR signaling played only a minor role in the development of Th2-dependent pathology in the lung, liver, and intestine against S. mansoni eggs [97]. Collectively, these findings suggest that although TSLPR signaling serves a key role in allergen-driven $\mathrm{Th} 2$ responses, it exerts only restricted regulatory activity during certain chronic helminth infections. Similar to TSLP, IL-25 is required for the development of a Th2 cytokine-mediated response and protective immunity following Trichuris or Nippostrongylus infection, as IL-25-deficient mice could not clear the 
infection or showed a delayed expulsion [98, 99]. At this stage, it is unclear whether IL-25 is induced only at the beginning-during the acute phase of infection - and how its activity is influenced by the immunoregulatory processes that take place during chronic and severe helminth infections. Little is known about IL-33 induction by helminths; thus far, its presence has only been described following infection with Trichuris, in which the expression peaked early during infection, indicating that IL-33 acts primarily during the initiation of these responses [100]. More studies must be carried out to evaluate the role of IL-33 in helminth infection, although initial studies favor a model in which IL-33 is induced early during infection and not so much during chronicallergy-protective-stages.

In helminth-infected or nonallergic, healthy individuals, $\mathrm{T}$ cells remain nonresponsive to harmless allergens, likely by actively created mucosal tolerance. New concepts are being introduced that suggest that mucosal tolerance to allergens may result from "tolerant" cross-talk between lung ECs and mucosal DCs. In this respect, lessons can be learned from the gut, in which intestinal ECs were found to drive the differentiation of Treg-promoting DCs via the expression of TGF- $\beta$ and retinoic acid [101]. Likewise, a similar role in homeostasis is suggested for lung ECs via promotion of anti-inflammatory and prophagocytic cells (eg, primary bronchial ECs induced macrophage differentiation from monocytes [102], whereas a lung epithelial cell line inhibited IL-12 and tumor necrosis factor- $\alpha$ production from antigen-presenting cells) [103]. Tolerance induction in the gut is strongly dependent on steady-state recognition of normal microbiota, as demonstrated in germ-free or knockout mice for specific TLRs, which recognize conserved microbial motifs [104]. Interestingly, molecules from several microorganisms could also induce IL-10 production in lung epithelial cell lines [105, 106]. These recent findings point toward a novel and exciting concept that microbial organisms can influence tolerance under homeostatic conditions by influencing cross-talk between ECs and other immune cells and pose the question of in which respect helminth species could interfere in the crosstalk of ECs and other immune cells.

\section{Therapeutic Application}

The case is now building that the epidemic rise in immunologic disorders in Westernized countries - for allergic diseases in particular - can be explained by a dysfunctional immune regulation resulting from decreased parasitic infections (eg, helminth infections). The question remains as to how this newly gained insight can help to stop the allergic March or to treat patients once they have become allergic. In search of novel therapies, it would be ideal to exploit the ability of chronic helminth infections to modulate the immune system. Promising results have been obtained by treating colitis patients with Trichuris suis, a whipworm that naturally infects pigs, and worm-based therapies are under development at several pharmaceutical companies [2]. Nevertheless, it would be more practical to focus on individual helminth-derived immunomodulatory molecules to selectively induce regulatory immune responses and avoid any possible side effects of natural worm infections. Some laboratories are isolating agents from helminths that could prove useful as therapeutic agents. These immunomodulatory parasite-derived compounds (detailed above) would form an ideal basis for therapeutic application in the treatment of hyperinflammatory disorders. However, one caveat in this immunoregulatory scenario is the possible interference with essential responses to other antigens, such as those contained in vaccinations, or to life-threatening pathogens. Therefore, serious efforts should be made to generate antigen-specific immunoregulation in order to circumvent dangerous side effects.

\section{Conclusions}

There is evidence that chronic, but not acute, helminth infection is driving responses that protect against allergic disorders. It is hypothesized that particularly during the course of chronic diseases, immunoregulatory processes are switched on (eg, the development of regulatory $\mathrm{T}$ and $\mathrm{B}$ cells, possibly via their priming by DCs, or innate signals from epithelial cells that have been in contact with certain signature immunomodulatory molecules). In view of these conclusions, it would make sense to focus on molecules that are expressed during the chronic phase of infection. A few molecules have been identified (detailed previously) that would make suitable candidates for therapy. It is important that efforts be made to bring these molecules to the clinic, preferably coupled to allergens to target allergenspecific changes and allow low concentrations to be effective.

Acknowledgments This work was supported by the Dutch Organization for Scientific Research grant no. ZONMW 912-03-048, ZONMW-VENI 016.066.093 (to Dr. Smits) and the European Commission contract no. EEG LSHB-CT-2006-018996 GABRIEL (to Dr. Everts).

Disclosure No potential conflicts of interest relevant to this article were reported.

Open Access This article is distributed under the terms of the Creative Commons Attribution Noncommercial License which permits any noncommercial use, distribution, and reproduction in any medium, provided the original author(s) and source are credited. 


\section{References}

1. Patel SP, Jarvelin MR, Little MP: Systematic review of worldwide variations of the prevalence of wheezing symptoms in children. Environ Health 2008, 7:57.

2. Weinstock JV, Elliott DE: Helminths and the IBD hygiene hypothesis. Inflamm Bowel Dis 2009, 15:128-133.

3. Schaub B, Lauener R, von Mutius E: The many faces of the hygiene hypothesis. J Allergy Clin Immunol 2006, 117:969-977.

4. Leonardi-Bee J, Pritchard D, Britton J: Asthma and current intestinal parasite infection: systematic review and meta-analysis. Am J Respir Crit Care Med 2006, 174:514-523.

5. Cooper PJ: Interactions between helminth parasites and allergy. Curr Opin Allergy Clin Immunol 2009, 9:29-37.

6. Cooper PJ, Barreto ML, Rodrigues LC: Human allergy and geohelminth infections: a review of the literature and a proposed conceptual model to guide the investigation of possible causal associations. Br Med Bull 2006, 79-80:203-218.

7. Rodrigues LC, Newcombe PJ, Cunha SS, et al.: Early infection with Trichuris trichiura and allergen skin test reactivity in later childhood. Clin Exp Allergy 2008, 38:1769-1777.

8. Guadalupe I, Mitre E, Benitez S, et al.: Evidence for in utero sensitization to Ascaris lumbricoides in newborns of mothers with ascariasis. J Infect Dis 2009, 199:1846-1850.

9. Djuardi Y, Wibowo H, Supali T, et al.: Determinants of the relationship between cytokine production in pregnant women and their infants. PLoS One 2009, 4:e7711.

10. Smits HH, Yazdanbakhsh M: Chronic helminth infections modulate allergen-specific immune responses: protection against development of allergic disorders? Ann Med 2007, 39:428-439.

11. Hartgers FC, Obeng BB, Kruize YC, et al.: Lower expression of TLR2 and SOCS-3 is associated with Schistosoma haematobium infection and with lower risk for allergic reactivity in children living in a rural area in Ghana. PLoS Negl Trop Dis 2008, 2:e227.

12. Obeng BB, Hartgers F, Boakye D, Yazdanbakhsh M: Out of Africa: what can be learned from the studies of allergic disorders in Africa and Africans? Curr Opin Allergy Clin Immunol 2008, 8:391-397.

13. Palmer LJ, Celedon JC, Weiss ST, et al.: Ascaris lumbricoides infection is associated with increased risk of childhood asthma and atopy in rural China. Am J Respir Crit Care Med 2002, 165:1489-1493.

14. Obihara CC, Beyers N, Gie RP, et al.: Respiratory atopic disease, Ascaris-immunoglobulin $\mathrm{E}$ and tuberculin testing in urban South African children. Clin Exp Allergy 2006, 36:640-648.

15. Moller M, Gravenor MB, Roberts SE, et al.: Genetic haplotypes of Th-2 immune signalling link allergy to enhanced protection to parasitic worms. Hum Mol Genet 2007, 16:1828-1836.

16. Flohr C, Quinnell RJ, Britton J: Do helminth parasites protect against atopy and allergic disease? Clin Exp Allergy 2009, 39:20-32.

17. Medeiros M Jr, Figueiredo JP, Almeida MC, et al.: Schistosoma mansoni infection is associated with a reduced course of asthma. J Allergy Clin Immunol 2003, 111:947-951.

18. Buijs J, Borsboom G, Renting M, et al.: Relationship between allergic manifestations and Toxocara seropositivity: a crosssectional study among elementary school children. Eur Respir J 1997, 10:1467-1475.

19. Gonzalez-Quintela A, Gude F, Campos J, et al.: Toxocara infection seroprevalence and its relationship with atopic features in a general adult population. Int Arch Allergy Immunol 2006, 139:317-324.

20. Cooper PJ, Chico ME, Vaca MG, et al.: Effect of albendazole treatments on the prevalence of atopy in children living in communities endemic for geohelminth parasites: a clusterrandomised trial. Lancet 2006, 367:1598-1603.
21. Lynch NR, Hagel I, Perez M, et al.: Effect of anthelmintic treatment on the allergic reactivity of children in a tropical slum. J Allergy Clin Immunol 1993, 92:404-411.

22. van den Biggelaar AH, Rodrigues LC, van Ree R, et al.: Longterm treatment of intestinal helminths increases mite skin-test reactivity in Gabonese schoolchildren. J Infect Dis 2004, 189:892-900.

23. Flohr C, Tuyen LN, Quinnell RJ, et al.: Reduced helminth burden increases allergen skin sensitization but not clinical allergy: a randomized, double-blind, placebo-controlled trial in Vietnam. Clin Exp Allergy 2009, 40(1):131-142.

24. Maizels RM, Yazdanbakhsh M: T-cell regulation in helminth parasite infections: implications for inflammatory diseases. Chem Immunol Allergy 2008, 94:112-123.

25. van Riet E, Retra K, Adegnika AA, et al.: Cellular and humoral responses to tetanus vaccination in Gabonese children. Vaccine 2008, 26:3690-3695.

26. van Riet E, Adegnika AA, Retra K, et al.: Cellular and humoral responses to influenza in Gabonese children living in rural and semi-urban areas. J Infect Dis 2007, 196:1671-1678.

27. Elias D, Britton S, Aseffa A, et al.: Poor immunogenicity of BCG in helminth infected population is associated with increased in vitro TGF-beta production. Vaccine 2008, 26:3897-3902.

28. Hartgers FC, Yazdanbakhsh M: Co-infection of helminths and malaria: modulation of the immune responses to malaria. Parasite Immunol 2006, 28:497-506.

29. Hartgers FC, Obeng BB, Kruize YC, et al.: Responses to malarial antigens are altered in helminth-infected children. J Infect Dis 2009, 199:1528-1535.

30. Wilson MS, Taylor MD, Balic A, et al.: Suppression of allergic airway inflammation by helminth-induced regulatory $\mathrm{T}$ cells. J Exp Med 2005, 202:1199-1212.

31. Kitagaki K, Businga TR, Racila D, et al.: Intestinal helminths protect in a murine model of asthma. J Immunol 2006, 177:1628-1635.

32. Hartmann S, Schnoeller C, Dahten A, et al.: Gastrointestinal nematode infection interferes with experimental allergic airway inflammation but not atopic dermatitis. Clin Exp Allergy 2009, 39(10):585-596.

33. Bashir ME, Andersen P, Fuss IJ, et al.: An enteric helminth infection protects against an allergic response to dietary antigen. J Immunol 2002, 169:3284-3292.

34. McConchie BW, Norris HH, Bundoc VG, et al.: Ascaris suumderived products suppress mucosal allergic inflammation in an interleukin-10-independent manner via interference with dendritic cell function. Infect Immun 2006, 74:6632-6641.

35. Araujo CA, Perini A, Martins MA, et al.: PAS-1, a protein from Ascaris suum, modulates allergic inflammation via IL-10 and IFN-gamma, but not IL-12. Cytokine 2008, 44:335-341.

36. Wohlleben G, Trujillo C, Muller J, et al.: Helminth infection modulates the development of allergen-induced airway inflammation. Int Immunol 2004, 16:585-596.

37. Smits HH, Hammad H, van Nimwegen M, et al.: Protective effect of Schistosoma mansoni infection on allergic airway inflammation depends on the intensity and chronicity of infection. J Allergy Clin Immunol 2007, 120:932-940.

38. Mangan NE, van Rooijen N, McKenzie AN, Fallon PG: Helminth-modified pulmonary immune response protects mice from allergen-induced airway hyperresponsiveness. J Immunol 2006, 176:138-147.

39. Pacifico LG, Marinho FA, Fonseca CT, et al.: Schistosoma mansoni antigens modulate experimental allergic asthma in a murine model: a major role for CD4+ CD25+ FoxP3+ T cells independent of interleukin-10. Infect Immun 2009, 77:98-107. 
40. Enobe CS, Araujo CA, Perini A, et al.: Early stages of Ascaris suum induce airway inflammation and hyperreactivity in a mouse model. Parasite Immunol 2006, 28:453-461.

41. Pinelli E, Brandes S, Dormans J, et al.: Infection with the roundworm Toxocara canis leads to exacerbation of experimental allergic airway inflammation. Clin Exp Allergy 2008, 38:649-658.

42. Strait RT, Morris SC, Smiley K, et al.: IL-4 exacerbates anaphylaxis. J Immunol 2003, 170:3835-3842.

43. Kreider T, Anthony RM, Urban JF Jr, Gause WC: Alternatively activated macrophages in helminth infections. Curr Opin Immunol 2007, 19:448-453.

44. Umetsu DT, DeKruyff RH: Immune dysregulation in asthma. Curr Opin Immunol 2006, 18:727-732.

45. Hartl D, Koller B, Mehlhorn AT, et al.: Quantitative and functional impairment of pulmonary $\mathrm{CD} 4+\mathrm{CD} 25 \mathrm{hi}$ regulatory $\mathrm{T}$ cells in pediatric asthma. J Allergy Clin Immunol 2007, 119:1258-1266.

46. Akdis M: Immune tolerance in allergy. Curr Opin Immunol 2009, 21(6):700-707.

47. Maizels RM, Yazdanbakhsh M: Immune regulation by helminth parasites: cellular and molecular mechanisms. Nat Rev Immunol 2003, 3:733-744.

48. Fallon PG, Mangan NE: Suppression of Th2-type allergic reactions by helminth infection. Nat Rev Immunol 2007, 7:220-230.

49. Satoguina J, Mempel M, Larbi J, et al.: Antigen-specific T regulatory-1 cells are associated with immunosuppression in a chronic helminth infection (onchocerciasis). Microbes Infect 2002, 4:1291-1300.

50. Doetze A, Satoguina J, Burchard G, et al.: Antigen-specific cellular hyporesponsiveness in a chronic human helminth infection is mediated by $\mathrm{T}(\mathrm{h}) 3 / \mathrm{T}(\mathrm{r}) 1$-type cytokines IL-10 and transforming growth factor-beta but not by a T(h)1 to T(h) 2 shift. Int Immunol 2000, 12:623-630.

51. Babu S, Blauvelt CP, Kumaraswami V, Nutman TB: Regulatory networks induced by live parasites impair both Th1 and Th2 pathways in patent lymphatic filariasis: implications for parasite persistence. J Immunol 2006, 176:3248-3256.

52. Watanabe K, Mwinzi PN, Black CL, et al.: T regulatory cell levels decrease in people infected with Schistosoma mansoni on effective treatment. Am J Trop Med Hyg 2007, 77:676-682.

53. Wammes LJ, Hamid F, Wiria AE, et al.: Regulatory T cells in human geohelminth infection suppress immune responses to BCG and Plasmodium falciparum. Eur J Immunol 2010, 40 (2):1-6.

54. Baumgart M, Tompkins F, Leng J, Hesse M: Naturally occurring CD4+Foxp3+ regulatory T cells are an essential, IL-10-independent part of the immunoregulatory network in Schistosoma mansoni egg-induced inflammation. J Immunol 2006, 176:5374-5387.

55. Huehn J, Siegmund K, Lehmann JC, et al.: Developmental stage, phenotype, and migration distinguish naive- and effector/memory-like CD4+ regulatory T cells. J Exp Med 2004, 199:303313.

56. McKee AS, Pearce EJ: CD25+CD4+ cells contribute to Th2 polarization during helminth infection by suppressing Th1 response development. J Immunol 2004, 173:1224-1231.

57. Taylor JJ, Mohrs M, Pearce EJ: Regulatory T cell responses develop in parallel to Th responses and control the magnitude and phenotype of the Th effector population. J Immunol 2006, 176:5839-5847.

58. Hesse M, Piccirillo CA, Belkaid Y, et al.: The pathogenesis of schistosomiasis is controlled by cooperating IL-10-producing innate effector and regulatory $\mathrm{T}$ cells. J Immunol 2004, 172:3157-3166

59. Sadler CH, Rutitzky LI, Stadecker MJ, Wilson RA: IL-10 is crucial for the transition from acute to chronic disease state during infection of mice with Schistosoma mansoni. Eur J Immunol 2003, 33:880888.
60. Fillatreau S, Gray D, Anderton SM: Not always the bad guys: B cells as regulators of autoimmune pathology. Nat Rev Immunol 2008, 8:391-397.

61. Lund FE: Cytokine-producing B lymphocytes-key regulators of immunity. Curr Opin Immunol 2008, 20:332-338.

62. Velupillai P, Secor WE, Horauf AM, Harn DA: B-1 cell $(\mathrm{CD} 5+\mathrm{B} 220+)$ outgrowth in murine schistosomiasis is genetically restricted and is largely due to activation by polylactosamine sugars. J Immunol 1997, 158:338-344.

63. Mangan NE, Fallon RE, Smith P, et al.: Helminth infection protects mice from anaphylaxis via IL-10-producing B cells. J Immunol 2004, 173:6346-6356.

64. Jankovic D, Cheever AW, Kullberg MC, et al.: CD4+ T cellmediated granulomatous pathology in schistosomiasis is downregulated by a $\mathrm{B}$ cell-dependent mechanism requiring $\mathrm{Fc}$ receptor signaling. J Exp Med 1998, 187:619-629.

65. Gillan V, Lawrence RA, Devaney E: B cells play a regulatory role in mice infected with the L3 of Brugia pahangi. Int Immunol 2005, $17: 373-382$

66. Correale J, Farez M, Razzitte G: Helminth infections associated with multiple sclerosis induce regulatory B cells. Ann Neurol 2008, 64:187-199.

67. Singh A, Carson WF, Secor ER Jr, et al.: Regulatory role of B cells in a murine model of allergic airway disease. J Immunol 2008, 180:7318-7326.

68. Wilson MS, Taylor MD, Balic A, et al.: Helminth-induced CD19+CD23hi B cells modulate experimental allergic and autoimmune inflammation. Eur J Immunol 2010 (in press).

69. Joffre O, Nolte MA, Sporri R, Reis e Sousa C: Inflammatory signals in dendritic cell activation and the induction of adaptive immunity. Immunol Rev 2009, 227:234-247.

70. Everts B, Perona-Wright G, Smits HH, et al.: Omega-1, a glycoprotein secreted by Schistosoma mansoni eggs, drives Th2 responses. J Exp Med 2009, 206:1673-1680.

71. Steinfelder S, Andersen JF, Cannons JL, et al.: The major component in schistosome eggs responsible for conditioning dendritic cells for Th2 polarization is a T2 ribonuclease (omega-1). J Exp Med 2009, 206:1681-1690.

72. Goodridge HS, McGuiness S, Houston KM, et al.: Phosphorylcholine mimics the effects of ES-62 on macrophages and dendritic cells. Parasite Immunol 2007, 29:127-137.

73. Thomas PG, Carter MR, Atochina O, et al.: Maturation of dendritic cell 2 phenotype by a helminth glycan uses a Tolllike receptor 4-dependent mechanism. J Immunol 2003, 171:5837-5841.

74. van Liempt E, van Vliet SJ, Engering A, et al.: Schistosoma mansoni soluble egg antigens are internalized by human dendritic cells through multiple C-type lectins and suppress TLR-induced dendritic cell activation. Mol Immunol 2007, 44:2605-2615.

75. van Riet E, Everts B, Retra K, et al.: Combined TLR2 and TLR4 ligation in the context of bacterial or helminth extracts in human monocyte derived dendritic cells: molecular correlates for Th1/ Th2 polarization. BMC Immunol 2009, 10:9.

76. Kane CM, Jung E, Pearce EJ: Schistosoma mansoni egg antigenmediated modulation of Toll-like receptor (TLR)-induced activation occurs independently of TLR2, TLR4, and MyD88. Infect Immun 2008, 76:5754-5759.

77. van der Kleij D, Latz E, Brouwers JF, et al.: A novel hostparasite lipid cross-talk. Schistosomal lyso-phosphatidylserine activates Toll-like receptor 2 and affects immune polarization. J Biol Chem 2002, 277:48122-48129.

78. Wang X, Zhou S, Chi Y, et al.: CD4(+)CD25(+) Treg induction by an HSP60-derived peptide SJMHE1 from Schistosoma japonicum is TLR2 dependent. Eur J Immunol 2009, 39:30523065. 
79. Layland LE, Rad R, Wagner H, da Costa CU: Immunopathology in schistosomiasis is controlled by antigen-specific regulatory $\mathrm{T}$ cells primed in the presence of TLR2. Eur J Immunol 2007, 37:2174 2184.

80. Sutmuller RP, den Brok MH, Kramer M, et al.: Toll-like receptor 2 controls expansion and function of regulatory $\mathrm{T}$ cells. J Clin Invest 2006, 116:485-494.

81. Liu H, Komai-Koma M, Xu D, Liew FY: Toll-like receptor 2 signaling modulates the functions of CD4+ CD25+ regulatory $\mathrm{T}$ cells. Proc Natl Acad Sci U S A 2006, 103:7048-7053.

82. van Die I, van Vliet SJ, Nyame AK, et al.: The dendritic cellspecific C-type lectin DC-SIGN is a receptor for Schistosoma mansoni egg antigens and recognizes the glycan antigen Lewis $\mathrm{x}$. Glycobiology 2003, 13:471-478.

83. Okano M, Satoskar AR, Nishizaki K, et al.: Induction of Th2 responses and $\mathrm{IgE}$ is largely due to carbohydrates functioning as adjuvants on Schistosoma mansoni egg antigens. J Immunol 1999, 163:6712-6717.

84. Okano M, Satoskar AR, Nishizaki K, Harn DA Jr: Lacto-Nfucopentaose III found on Schistosoma mansoni egg antigens functions as adjuvant for proteins by inducing Th2-type response. J Immunol 2001, 167:442-450.

85. Schabussova I, Amer H, van Die I, et al.: O-methylated glycans from Toxocara are specific targets for antibody binding in human and animal infections. Int J Parasitol 2007, 37:97-109.

86. Tawill S, Le Goff L, Ali F, et al.: Both free-living and parasitic nematodes induce a characteristic $\mathrm{Th} 2$ response that is dependent on the presence of intact glycans. Infect Immun 2004, 72:398-407.

87. Silva SR, Jacysyn JF, Macedo MS, Faquim-Mauro EL: Immunosuppressive components of Ascaris suum down-regulate expression of costimulatory molecules and function of antigenpresenting cells via an IL-10-mediated mechanism. Eur J Immunol 2006, 36:3227-3237.

88. Perona-Wright G, Jenkins SJ, MacDonald AS: Dendritic cell activation and function in response to Schistosoma mansoni. Int J Parasitol 2006, 36:711-721.

89. Carvalho L, Sun J, Kane C, et al.: Review series on helminths, immune modulation and the hygiene hypothesis: mechanisms underlying helminth modulation of dendritic cell function. Immunology 2009, 126:28-34.

90. Geijtenbeek TB, Gringhuis SI: Signalling through C-type lectin receptors: shaping immune responses. Nat Rev Immunol 2009, 9:465-479.

91. Ferret-Bernard S, Curwen RS, Mountford AP: Proteomic profiling reveals that Th2-inducing dendritic cells stimulated with helminth antigens have a 'limited maturation' phenotype. Proteomics 2008, 8:980-993.

92. Saenz SA, Taylor BC, Artis D: Welcome to the neighborhood: epithelial cell-derived cytokines license innate and adaptive immune responses at mucosal sites. Immunol Rev 2008, 226:172190.

93. Barrett NA, Austen KF: Innate cells and T helper 2 cell immunity in airway inflammation. Immunity 2009, 31:425-437.

94. Rochman Y, Leonard WJ: Thymic stromal lymphopoietin: a new cytokine in asthma. Curr Opin Pharmacol 2008, 8:249-254.

95. Zaph C, Troy AE, Taylor BC, et al.: Epithelial-cell-intrinsic IKK-beta expression regulates intestinal immune homeostasis. Nature 2007, 446:552-556.

96. Massacand JC, Stettler RC, Meier R, et al.: Helminth products bypass the need for TSLP in Th2 immune responses by directly modulating dendritic cell function. Proc Natl Acad Sci U S A 2009, 106:13968-13973.

97. Ramalingam TR, Pesce JT, Mentink-Kane MM, et al.: Regulation of helminth-induced Th2 responses by thymic stromal lymphopoietin. J Immunol 2009, 182:6452-6459.

98. Owyang AM, Zaph C, Wilson EH, et al.: Interleukin 25 regulates type 2 cytokine-dependent immunity and limits chronic inflammation in the gastrointestinal tract. J Exp Med 2006, 203:843-849.

99. Fallon PG, Ballantyne SJ, Mangan NE, et al.: Identification of an interleukin (IL)-25-dependent cell population that provides IL-4, IL-5, and IL-13 at the onset of helminth expulsion. J Exp Med 2006, 203:1105-1116.

100. Humphreys NE, Xu D, Hepworth MR, et al.: IL-33, a potent inducer of adaptive immunity to intestinal nematodes. J Immunol 2008, 180:2443-2449.

101. Iliev ID, Mileti E, Matteoli G, et al.: Intestinal epithelial cells promote colitis-protective regulatory $\mathrm{T}$-cell differentiation through dendritic cell conditioning. Mucosal Immunol 2009, 2:340-350.

102. Ward C, Eger K, Diboll J, et al.: Bronchial epithelial cells cultured from clinically stable lung allograft patients promote the development of macrophages from monocytes rather than dendritic cells. Thorax 2009, 64:430-435.

103. Mayer AK, Bartz H, Fey F, et al.: Airway epithelial cells modify immune responses by inducing an anti-inflammatory microenvironment. Eur J Immunol 2008, 38:1689-1699.

104. Wells JM, Loonen LM, Karczewski JM: The role of innate signaling in the homeostasis of tolerance and immunity in the intestine. Int $\mathbf{J}$ Med Microbiol 2010, 300(1):41-48.

105. Mendez-Samperio P, Trejo A, Perez A: Mycobacterium bovis Bacillus Calmette-Guerin (BCG) stimulates IL-10 production via the PI3K/Akt and p38 MAPK pathways in human lung epithelial cells. Cell Immunol 2008, 251:37-42.

106. Pichavant M, Taront S, Jeannin P, et al.: Impact of bronchial epithelium on dendritic cell migration and function: modulation by the bacterial motif KpOmpA. J Immunol 2006, 177:59125919 . 